\title{
Kupffer cell depletion by gadolinium chloride aggravates liver injury after brain death in rats
}

\author{
RONGTAO ZHU ${ }^{1}$, WEIZHI GUO ${ }^{1,2}$, HONGBO FANG $^{1,2}$, SHENGLI CAO $^{1,2}$, BING YAN $^{1,2}$, \\ SANYANG CHEN $^{1,2}$, KAIMING ZHANG ${ }^{1}$ and SHUIJUN ZHANG ${ }^{1,2}$ \\ ${ }^{1}$ Department of Hepatobiliary and Pancreatic Surgery; ${ }^{2}$ Key Laboratory of \\ Hepatobiliary and Pancreatic Surgery and Digestive Organ Transplantation of Henan Province, \\ The First Affiliated Hospital of Zhengzhou University, Zhengzhou, Henan 450052, P.R. China
}

Received October 5, 2017; Accepted February 8, 2018

DOI: $10.3892 / \mathrm{mmr} .2018 .8646$

\begin{abstract}
Brain death (BD) impairs liver function in potential donors, and is associated with hormonal and metabolic changes or molecular effects with pro-inflammatory activation. Resident macrophages in the liver named Kupffer cells (KCs) undergo pro- or anti-inflammatory pathway activation, which affects liver function. However, the role of the KCs in liver dysfunction following BD has not been fully elucidated. The aim of the present study was to investigate the role of $\mathrm{KCs}$ in liver dysfunction in the context of BD and the effects of their inhibition by gadolinium chloride $\left(\mathrm{GdCl}_{3}\right)$. Rats were randomly divided into the following groups: Control, BD with $\mathrm{GdCl}_{3}$ pretreatment and $\mathrm{BD}$ with normal saline pretreatment. Liver function, hepatic pathological histology and cytokine levels in the liver were assessed. Apoptosis and apoptosis-related proteins [cleaved caspase-3, caspase-3 and apoptosis regulator $\mathrm{Bcl}-2$ ( $\mathrm{Bcl}-2)$ ] were evaluated. $\mathrm{GdCl}_{3}$ significantly aggravated liver injury by elevating alanine aminotransferase and aspartate aminotransferase levels $(\mathrm{P}<0.05)$ by inhibiting KCs. Interleukin (IL) $-1 \beta$ and tumor necrosis factor $\alpha$ levels in the $\mathrm{GdCl}_{3}$ group were significantly increased compared with those in the control and saline groups $(\mathrm{P}<0.01)$. However, IL-10 levels in the $\mathrm{GdCl}_{3}$ group were significantly reduced compared with those in the saline group $(\mathrm{P}<0.05)$. Caspase- 3 and cleaved caspase- 3 activation, and apoptosis induction in the context of $\mathrm{BD}$ were also significantly aggravated by the depletion of $\mathrm{KCs}$,
\end{abstract}

Correspondence to: Professor Shuijun Zhang, Department of Hepatobiliary and Pancreatic Surgery, The First Affiliated Hospital of Zhengzhou University, 1 East Jianshe Road, Zhengzhou, Henan 450052, P.R. China

E-mail: zhangshuijun@zzu.edu.cn

Abbreviations: $\mathrm{KCs}$, Kupffer cells; $\mathrm{GdCl}_{3}$, gadolinium chloride; $\mathrm{BD}$, brain death; ALT, alanine aminotransferase; AST, aspartate aminotransferase; IL-1 $\beta$, interleukin-1 $\beta$; IL-10, interleukin-10; TNF- $\alpha$, tumor necrosis factor- $\alpha$

Key words: Kupffer cells, gadolinium chloride, liver injury, brain death whereas Bcl-2 was significantly suppressed by the administration of $\mathrm{GdCl}_{3}$. The present study indicated that $\mathrm{GdCl}_{3}$ efficiently inhibits the activity of $\mathrm{KCs}$, and is involved in the onset of liver injury through its effects on pro-inflammatory and anti-inflammatory activation. KCs are protective in the liver in the context of BD. This protection appears to be due to KCs secretion of the potent anti-inflammatory cytokine IL-10, suggesting that $\mathrm{KCs}$ are an attractive target for the prevention and treatment of liver injury in the context of BD in rats.

\section{Introduction}

An increasing imbalance between the number of donor organs and potential liver transplant recipients has led to the development of novel strategies to increase the pool of organ donors (1). The declaration of brain death (BD) as a point of no return has been accepted by most societies, and organs derived from BD donors currently represent the main source of organs used in transplantation $(1,2)$. Since the first BD donor organ transplantation in the 1960s, clinical transplant results of donor organs have significantly improved (3). To date, the grafts obtained from BD donors have yielded positive outcomes. However, $\mathrm{BD}$ is a dynamic and rather unphysiological course of events that influences a number of physiological processes in the human body, whereby potential grafts are damaged before liver transplantation (4). Several experiments demonstrated that systemic and hormonal changes occur immediately under BD conditions, followed by oxidative stress, an inflammatory response, and tissue ischemia reperfusion, all of which result in liver damage (5-8). BD itself has a complicated influence on the liver, thus limiting the number of suitable organs for liver transplantation (6-9). The mechanism underlying the deteriorating effect of $\mathrm{BD}$ on organs has not been fully established. Therefore, it is necessary to investigate the characterization of liver injury under conditions of BD to improve the outcome of liver transplantation.

As the resident macrophages in the liver, Kupffer cells (KCs) express key renin angiotensin system (RAS) components, and RAS activity potentially participates in pathology and physiology (10). The activation of donor KCs is closely correlated with intense phagocytosis, a high expression of membranous molecules, antigen presentation, and the secretion 
of numerous cytokines, which all participate in the immune and pro-inflammatory or anti-inflammatory reactions (11). However, the exact role of KCs in liver injury under conditions of BD remains unknown. The purpose of this study was to explore the role of KCs in inflammation and apoptosis in liver injury under conditions of BD in a rat model.

\section{Materials and methods}

Experimental animals and treatment. Sprague-Dawley (SD) rats weighing 300-350 g were purchased from Henan Provincial Experimental Animal Center (Zhengzhou, China). The Ethics Committee of the First Affiliated Hospital of Zhengzhou University (Zhengzhou, China) approved this study protocol. All animals were provided humane care in compliance with governmental regulations and institutional guidelines. In total, 24 rats were randomly divided into four groups as follows: Rats that underwent sham operations (Sham group); rats subjected to $\mathrm{BD}$ (BD group); rats subjected to $\mathrm{BD}$ plus gadolinium chloride $\left(\mathrm{GdCl}_{3}\right)$ treatment ( $\mathrm{Gd}$ group) with $\mathrm{GdCl}_{3}$ solution $(7 \mathrm{mg} / \mathrm{kg}$ of body weight delivered intraperitoneally; Sigma-Aldrich; Merck KGaA, Darmstadt, Germany) administered continuously for two days before the operation (12); and rats subjected to BD plus normal saline (Saline group). All operative procedures were the same as those described for the Gd group. Rats were sacrificed $6 \mathrm{~h}$ after BD, and 6 rats per time point were assessed.

Construction of BD models. The BD model was established according to previously reported methods (13-15). Rats were anesthetized via intraperitoneal injection of pentobarbital sodium (50 mg/kg body weight). The BD model was induced by gradually increasing intra-cranial pressure by slow inflation through a No. 3 Fogarty catheter balloon (Edwards Lifesciences Corp., Irvine, CA, USA) (13). Sham-operated rats underwent the same surgical procedures but without the induction of BD; this group served as BD controls. The rats were humanely sacrificed by cervical dislocation at the indicated time after BD as assessed by respiratory and circulatory parameters. Blood from the caudal vein and liver samples were harvested in situ and stored in liquid nitrogen until further analyses.

Analysis of histopathological changes and plasma markers of liver function. The liver tissues were fixed in $100 \mathrm{~g} / \mathrm{l}$ of neutral formalin solution and embedded in paraffin wax. The sections were stained with hematoxylin and eosin to assess morphological changes. Portal inflammation, periportal/bridging necrosis, intralobular degeneration/focal necrosis and fibrosis were evident pathological changes in the injured livers as scored by the Knodell histological activity index (16). The sections were evaluated in a blinded manner under light microscopy by two investigators. The necro-inflammatory score (HAI-NI) was analyzed to evaluate the severity of hepatic damage. Changes in plasma markers of liver function, serum alanine aminotransferase (ALT) and aspartate aminotransferase (AST) were detected using an automatic biochemical analyzer. The AST/ALT ratio (AAR) was measured to appraise liver damage following BD. Blood was collected through the caudal vein to measure ALT and AST levels.
ELISA for the analysis of liver cytokines. Total protein was extracted from hepatic tissue, and protein levels were normalized to measure the expression levels of tumor necrosis factor (TNF)- $\alpha$, interleukin (IL)-1 $\beta$ and IL-10. These protein levels were determined using rat TNF- $\alpha$, IL- $1 \beta$ and IL-10 ELISA kits (Wuhan Boster Biological Technology, Ltd., Wuhan, China) following the manufacturer's instructions. Sham-operated rats served as BD controls. The results are presented at each time point.

Apoptosis measurements. The concentration of apoptotic cells was determined using the terminal deoxynucleotidyl transferase dUTP nick end-labeling (TUNEL) assay. Apoptotic cells in liver tissue sections ( $4 \mu \mathrm{m})$ were identified using the in situ cell death detection kit (Fluorescein; Roche Diagnostics $\mathrm{GmbH}$, Mannheim, Germany) according to the manufacturer's protocol. The apoptotic index (AI) results are expressed as the percentage of apoptotic cells among the total number of cells.

Western blot analysis of liver tissue. Liver tissue samples were used to measure the expression levels of apoptosis-related proteins (cleaved caspase-3, caspase-3 and Bcl-2) using selective polyclonal antibodies. Protein concentrations of homogenates were determined using the Bradford technique. Specific bands were detected using an enhanced chemiluminescence system and captured on X-ray film. $\beta$-actin was used as a loading control. The density of the bands on the membrane was analyzed using Quantity One software v4.62 (Bio-Rad Laboratories, Inc., Hercules, CA, USA).

Data analysis. All values are expressed as the mean \pm standard deviation. The means of two continuous normally distributed variables were compared by independent samples Student's t-test. Multigroup comparisons of the means were performed using one-way analysis of variance with post hoc Student-Newman-Keuls test. Values were analyzed using the statistical package SPSS for Windows version 15.0 (SPSS, Inc., Chicago, IL, USA). Statistical significance was set at an $\alpha$ value of $\mathrm{P}=0.05$.

\section{Results}

$K C$ depletion aggravates liver injury under conditions of $B D$ Histopathological changes in the liver. Liver histological sections were stained with $\mathrm{H} \& \mathrm{E}$, and liver injury was graded according to the degree of necro-inflammation (HAI-NI) using the Knodell score system $(16,17)$. We observed that the zones of necrosis were located around pericentral areas in the rats, with the exception of rats undergoing sham operation. The hepatocytes in the $\mathrm{GdCl}_{3}$ group exhibited aggravated vacuolar degeneration and edema, and a greater number of inflammatory cells infiltrated the portal area compared with the B and S groups. However, there were no differences in injury between the saline group and the BD group. Saline had no effect on hepatocyte injury under conditions of BD (Fig. 1). The pathological Knodell scores of the livers exposed to $\mathrm{GdCl}_{3}$ treatment were significantly increased compared with those of the $\mathrm{B}$ and $\mathrm{S}$ groups $(\mathrm{P}<0.05)$. However, no significant difference was noted between the $\mathrm{B}$ and $\mathrm{S}$ groups $(\mathrm{P}>0.05)$. 

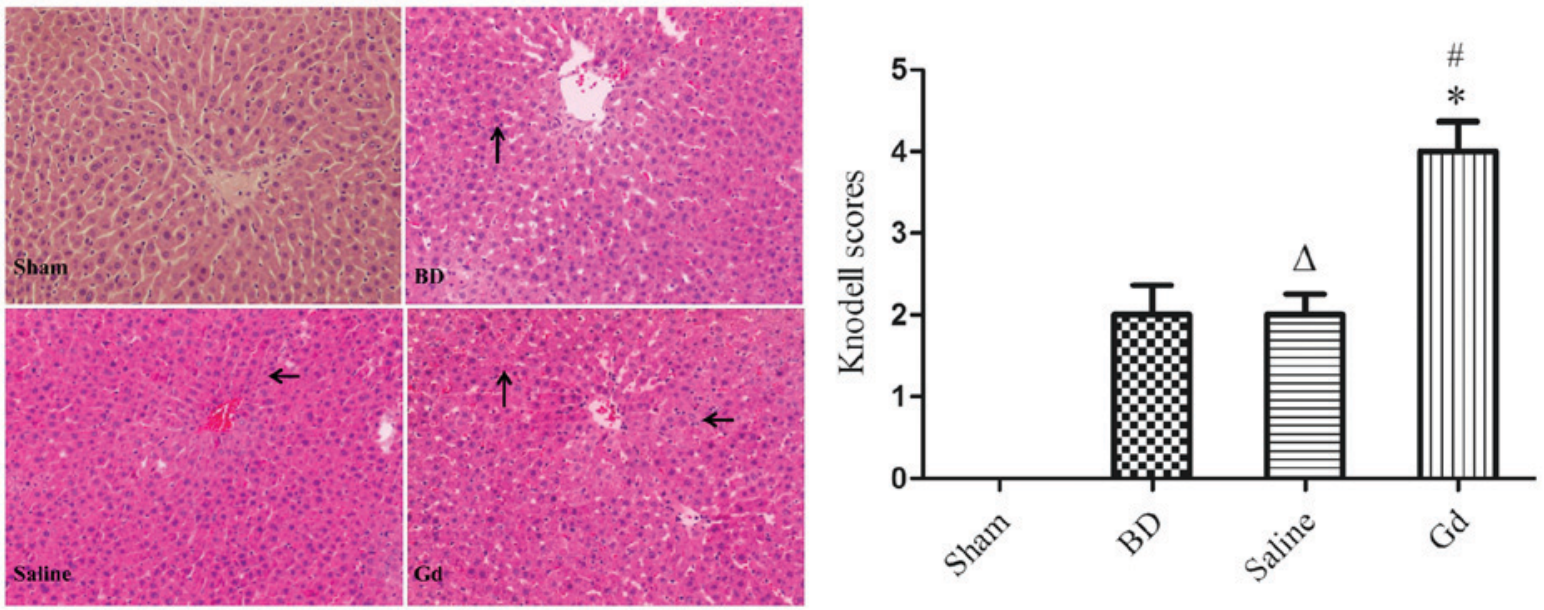

Figure 1. Liver injury following BD. Pretreatment of rats with $\mathrm{GdCl}_{3}$ aggravates liver injury following BD. Liver pathological microsections from each model rat were scored according to hepatocyte necrosis, inflammatory cell infiltration in lobules, vascular endothelial injury and thrombus formation (arrow). Scale bar $=50 \mu \mathrm{M}$. Knodell scores are expressed as the mean \pm standard deviation. ${ }^{\#}$ and ${ }^{*} \mathrm{P}<0.05$ vs. the BD group and the Saline group. ${ }^{\Delta} \mathrm{P}>0.05$ vs. the BD group. $\mathrm{BD}$, brain dead; $\mathrm{GdCl}_{3}$, gadolinium chloride.

Plasma liver function markers. After $6 \mathrm{~h}$ of BD, ALT levels increased 4-fold compared with those in the sham operation group. AST levels followed the same trend as ALT levels. Six h after BD, pretreatment with $\mathrm{GdCl}_{3}$ caused increases in ALT and AST levels compared with those in the saline group $(\mathrm{P}<0.05)$. However, pretreatment with saline had no effect on ALT and AST levels. Statistically significant differences in serum ALT and AST levels were noted between the saline and BD groups. $\mathrm{GdCl}_{3}$ treatment prior to BD increased AST/ALT ratio without significant statistical difference ( $\mathrm{P}>0.05$; Fig. 2). These results reveal that $\mathrm{GdCl}_{3}$ treatment significantly aggravated hepatocyte injury as assessed by ALT and AST levels and necrotic cell death without significant statistical difference of AAR.

\section{Effect of KCs on the regulation of liver inflammation}

ELISA results of liver pro-inflammatory cytokines. To more fully investigate the damaged phenotype displayed in $\mathrm{GdCl}_{3}$-treated animals subjected to $\mathrm{BD}$, the expression levels of liver pro-inflammatory cytokines were measured. In terms of the ELISA analyses of TNF- $\alpha$, IL- $1 \beta$ and IL-10, the concentrations of TNF- $\alpha$ and IL- $1 \beta$ in the $\mathrm{GdCl}_{3}$ group were markedly increased compared with those in the saline and $\mathrm{BD}$ groups $(\mathrm{P}<0.05)$. TNF- $\alpha$ and IL-1 $\beta$ levels were upregulated at $6 \mathrm{~h}$ in $\mathrm{GdCl}_{3}$-treated animals (Fig. 2).

ELISA results of liver anti-inflammatory cytokines. IL-10 was one of the most potent anti-inflammatory cytokines produced in the liver; therefore, IL-10 liver expression levels under BD conditions were measured. Regarding the ELISA analysis, a marked increase in IL-10 levels was noted in the saline and $\mathrm{BD}$ groups $6 \mathrm{~h}$ after BD compared with those in the sham operation group $(\mathrm{P}<0.05$; Fig. 2$)$. In contrast, IL-10 levels in the $\mathrm{GdCl}_{3}$-treated rats remained at baseline levels compared with those in the sham operation group $6 \mathrm{~h}$ after $\mathrm{BD}$. These results reveal the lack of IL-10 production in $\mathrm{GdCl}_{3}$-treated animals. IL-10 expression in the liver was significantly suppressed in the $\mathrm{GdCl}_{3}$-treated groups compared with the saline and BD groups $(\mathrm{P}<0.05$; Fig. 2).
Effect of KC depletion on liver cell apoptosis under BD conditions Liver apoptosis. Apoptotic cells were quantified in each field. In situ labeling of cell nuclei using the TUNEL assay revealed that BD significantly increased hepatic cell apoptosis in the saline and BD groups $6 \mathrm{~h}$ after BD compared with that in the sham operation group. The AI increased $6 \mathrm{~h}$ after BD. However, hepatic cell apoptosis was dramatically aggravated $6 \mathrm{~h}$ after $\mathrm{GdCl}_{3}$ treatment $(\mathrm{P}<0.05$; Fig. 3).

Western blot analysis of apoptosis-related proteins in liver tissue. To determine the importance of hepatic cell apoptosis in $\mathrm{KC}$-mediated protective mechanisms and BD-induced hepatic injury, western blot analysis of apoptosis-related proteins was performed on total hepatic protein. Caspase-3, cleaved caspase- 3 and Bcl-2 expression levels were examined by western blot analysis. Caspase- 3 and cleaved caspase- 3 expression levels were significantly increased, whereas Bcl-2 expression was significantly decreased 2 and $6 \mathrm{~h}$ after BD compared with those in the sham operation group $(\mathrm{P}<0.05)$. After $\mathrm{GdCl}_{3}$ treatment, caspase-3 and cleaved caspase-3 expression levels were significantly decreased, whereas Bcl-2 expression was significantly increased compared with those in the saline and BD groups $(\mathrm{P}<0.05 ;$ Fig. 4$)$.

\section{Discussion}

As resident macrophages of the liver, $\mathrm{KCs}$ excrete significant amounts of pro-inflammatory and anti-inflammatory cytokines and play a central role in the detrimental effects of liver transplantation. Studies have demonstrated that inhibition of $\mathrm{KC}$ activation through pharmacological mechanisms improved the outcomes of liver transplantation. Although different categories of hepatic macrophages were suppressed by liposomal clodronate to varying degrees, $\mathrm{KCs}$ were most susceptible to suppression $(12,18)$. Therefore, $\mathrm{GdCl}_{3}$ was used to inhibit $\mathrm{KC}$ function in our study. Several scholars reported that KCs were protective in liver tissues subjected to total ischemia/reperfusion (I/R) in transplantation (19). KCs are directly involved in inducing liver transplantation tolerance and hepatic I/R injury. 

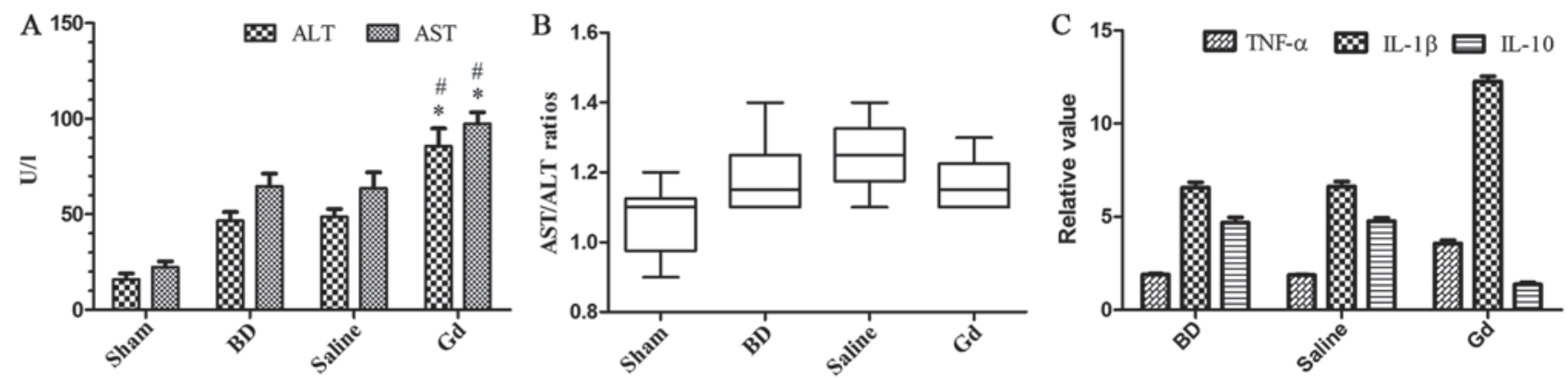

Figure 2. (A) ALT and AST levels in each group. $\mathrm{GdCl}_{3}$ treatment prior to $\mathrm{BD}$ increased hepatocellular damage. The data are expressed as the means \pm standard deviation (SD). "and " $\mathrm{P}<0.05$ vs. the BD group, saline group and sham group. (B) AST/ALT ratios in each group. GdCl ${ }_{3}$ treatment prior to BD increased AST/ALT ratio without significant statistical difference $(\mathrm{P}>0.05)$. (C) Inflammatory cytokine expression in liver. The results are presented as the fold change after normalization to baseline of the sham operation group, and the data are expressed as the means \pm SD. TNF- $\alpha$, IL-1 $\beta$ and IL-10 levels were upregulated in each liver group after BD. TNF and IL-1 $\beta$ levels were upregulated in $\mathrm{GdCl}_{3}$-treated animals vs. diluent-treated controls and the BD group, whereas IL-10 levels were significantly downregulated in $\mathrm{GdCl}_{3}$-treated animals vs. diluent-treated controls and the $\mathrm{BD}$ group. No significant differences were noted between the diluent-treated controls and the BD group. ALT, alanine aminotransferase; AST, aspartate aminotransferase; IL, interleukin; BD, brain dead; GdCl3, gadolinium chloride; TNF- $\alpha$, tumor necrosis factor- $\alpha$.

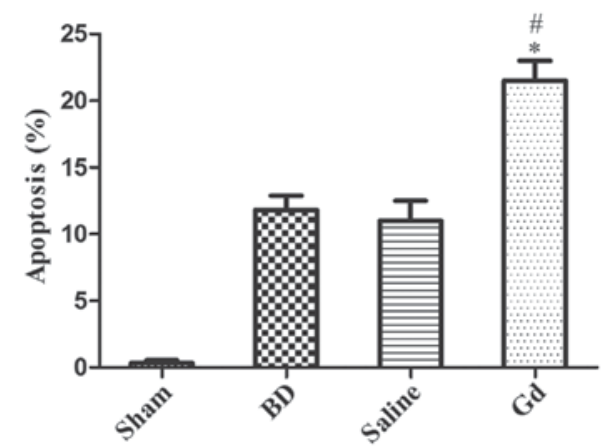

Figure 3. Liver AI. The AI is expressed as the percentage of apoptotic cells among the total number of cells. The level of apoptosis was increased in $\mathrm{GdCl}_{3}$-treated animals compared with those in the diluent-treated controls and the BD group. No significant differences were noted between the diluent-treated controls and the BD group. ${ }^{*}$ and ${ }^{~} \mathrm{P}<0.05$ vs. the $\mathrm{BD}$ group, saline group and sham group. AI, apoptotic index; $\mathrm{BD}$, brain dead; $\mathrm{GdCl}$, gadolinium chloride.

Furthermore, KCs are activated during the onset of $\mathrm{BD}$ induction in animals (6). Upon $\mathrm{GdCl}_{3}$ treatment to deplete $\mathrm{KCs}$, we demonstrated that extensive $\mathrm{KC}$ elimination aggravated liver injury after BD in rats. The levels of the pro-inflammatory cytokines TNF- $\alpha$ and IL-1 $\beta$ increased, whereas expression of the anti-inflammatory cytokine IL-10 in the liver decreased. In addition, liver cell apoptosis was accelerated by suppression of KCs. Our finding suggested that KCs play a protective role in liver injury after BD potentially based on IL-10 expression and liver apoptosis suppression.

Secondary to BD, inflammation is driven by both the innate and adaptive immune systems in addition to numerous cytokines (19). Studies have demonstrated that BD is associated with significant upregulation of inflammatory cytokines through several mechanisms, thus promoting severe injury after liver transplantation $(20,21)$. Upon methylprednisolone treatment, soluble ILs and TNF- $\alpha$ were significantly decreased, which significantly ameliorated liver injury post-transplantation (22).

IL-1 $\beta$ is an important mediator of the pro-inflammatory response and is produced by activated macrophages as a proprotein $(6,23)$. Research has demonstrated that $\mathrm{BD}$ promotes the induction of IL- $1 \beta$ and TNF- $\alpha$ based on the activation of non-parenchymal cells in the liver $(6,24)$. IL-10 suppresses the production of pro-inflammatory cytokines and upregulates inhibitors of IL-1 $\beta$ and TNF- $\alpha$, leading to impairment or reversal of the effects of pro-inflammatory mediators (25). In the liver under BD conditions, IL-10 was primarily produced by KCs, as reported previously (26). We observed that IL-1 $\beta$ and IL-10 were significantly increased in the liver tissues of BD rats. IL- $1 \beta$ and TNF- $\alpha$ expression in the liver increases $6 \mathrm{~h}$ after $\mathrm{BD}$, whereas $\mathrm{GdCL}_{3}$ treatment increased IL- $1 \beta$ and TNF- $\alpha$ levels. However, IL-10 was significantly decreased upon treatment with $\mathrm{GdCL}_{3}$. The data indicate that in the absence of $\mathrm{KCs}$, the production of liver cytokines was significantly imbalanced. We suggest that the absence of KCs and their production of IL-10 lead to a pro-inflammatory response under BD conditions, which aggravates liver injury after $\mathrm{BD}$ in rats.

Previous studies have demonstrated that BD induces hepatocellular apoptosis and liver dysfunction $(9,12,27)$. Therefore, we examined the effect of KCs on liver apoptosis upon treatment with $\mathrm{GdCl}_{3}$ under BD conditions. Caspase-3 is a major executioner caspase that is cleaved at an aspartate residue to become activated. Cleaved caspase- 3 degrades multiple cellular proteins and is responsible for morphological changes in cells during apoptosis. Previous research has demonstrated that apoptosis was obviously regulated by cleaved caspase- 3 and $\mathrm{Bcl}-2$ protein expression. Further studies revealed that the administration of the pan-caspase inhibitor IDN-6556 during liver transplantation offers local therapeutic protection against hepatocellular apoptosis and liver injury (28). Our present supporting evidence demonstrates that $\mathrm{KC}$ function is inhibited by $\mathrm{GdCl}_{3}$ treatment, shifting the balance of pro-apoptotic and anti-apoptotic molecules to favor cell death. We observed that BD induces hepatocellular apoptosis, upregulation of cleaved caspase-3 expression and suppression of Bcl-2 expression. Under conditions of $\mathrm{GdCl}_{3}$ pretreatment, increased liver apoptosis was noted with increased cleaved caspase-3 and caspase-3 expression and decreased Bcl-2 expression. KC elimination by $\mathrm{GdCl}_{3}$ aggravated liver injury in a manner dependent on the regulation of apoptosis under BD conditions in rats by shifting the balance of cleaved caspase- 3 and Bcl-2 molecules to favor cell death. Therefore, KCs may protect 

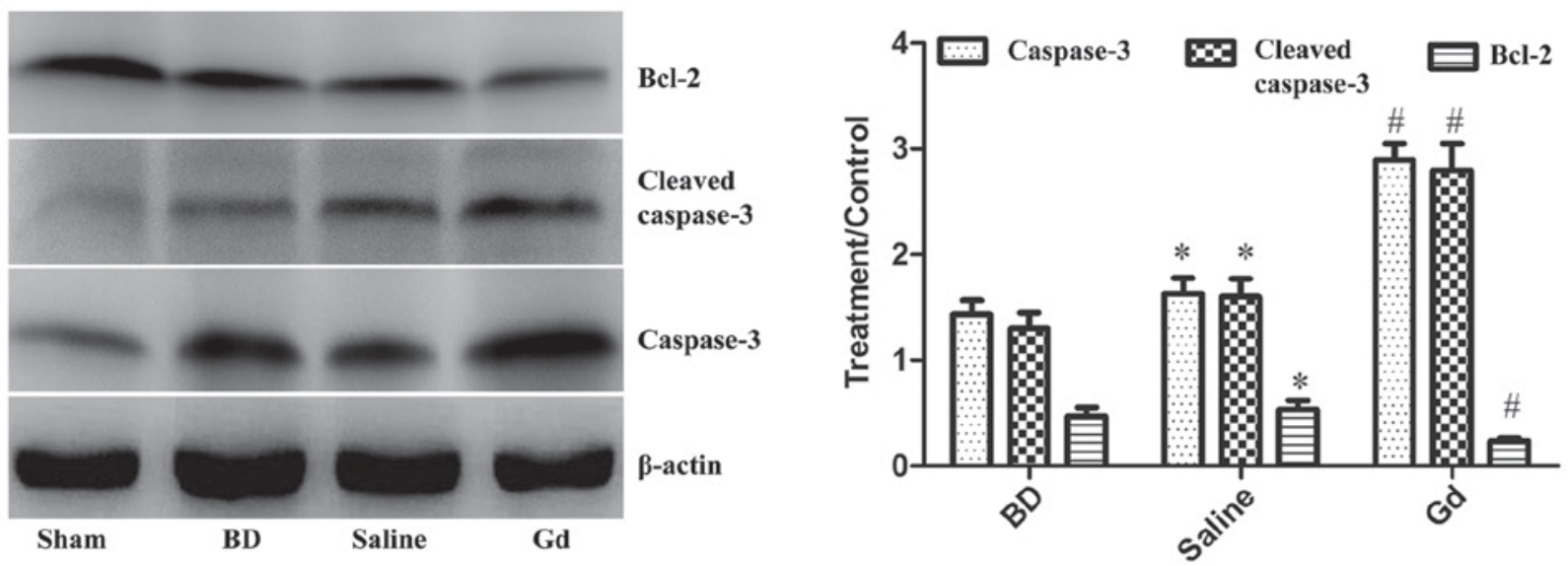

Figure 4. Western blot analysis of apoptosis-related proteins in liver tissue. Each protein band was quantified and normalized to $\beta$-actin, and the results of every group are presented as the fold change after normalization to the baseline of the sham operation group. ${ }^{*} \mathrm{P}<0.05$ vs. the BD and saline groups. ${ }^{~} \mathrm{P}>0.05$ vs. the BD group. BD, brain dead.

against BD by inhibiting hepatocyte apoptosis. Similarly, caspase-3 and cleaved caspase-3 expression increased, and $\mathrm{Bcl}-2$ expression decreased. Given that caspase-3 promotes apoptosis and $\mathrm{Bcl}-2$ impedes apoptosis, $\mathrm{KCs}$ may regulate cleaved caspase-3 and Bcl-2 to fight against apoptosis in BD. In summary, these results provide strong evidence that $\mathrm{KCs}$ protect liver function under BD conditions through the regulation of a pro-inflammatory state and apoptosis.

Murine KCs are protective in total hepatic I/R injury $(19,29)$, and these results were similar to those presented in our research. KCs are exceptionally plastic cells that can polarize to specific activation states and perform various functions in different microenvironments. It has been reported that alternatively activated M2 KCs can promote caspase-3-dependent apoptosis of classically activated M1 KCs, thus providing a protective mechanism. The M2-mediated apoptosis of M1 KCs was shown to be arginase dependent by way of IL-10 (30). Another study demonstrated that $\mathrm{GdCl}_{3}$ played an important protective role in early I/R injury and suppressed bile duct cell apoptosis during liver transplantation (31). During BD, liver-specific parameters of plasma endotoxin levels and the endotoxin-neutralizing capacity were compromised independent of hemodynamic status (32). Despite varying inflammatory profiles in organs after $\mathrm{BD}, \mathrm{BD}$ does not accelerate $\mathrm{I} / \mathrm{R}$ injury in transplantation (33). This discrepant finding is likely due to the predominant inflammatory component resulting from plasma endotoxin levels in our BD model, which differs from I/R injury. In this study, we demonstrated that KCs appear to play a protective role in liver subjected to BD. No single method is available to inhibit apoptosis and apoptosis genes to verify the role of KCs in liver injury protection in the context of $\mathrm{BD}$. Therefore, the protective effects of KCs in liver injury observed in this study may only represent one aspect of the overall protective mechanism. In addition, it may be concluded that the effect of KCs in liver injury under conditions of $\mathrm{BD}$ involves a combined action requiring the participation of numerous mechanisms. Further studies may also be necessary to understand the exact mechanism.

In summary, our study indicates that KCs play a protective role in livers subjected to $\mathrm{BD}$, which appears to be due to $\mathrm{KC}$ secretion of the potent anti-inflammatory cytokine IL-10. We also demonstrated that $\mathrm{GdCl}_{3}$ efficiently inhibits the activity of
KCs, which participate in the onset of liver injury through its effect on pro-inflammatory and anti-inflammatory activation. All of these results suggest that KCs are a potential target of $\mathrm{GdCl}_{3}$ for the prevention and treatment of liver injury under $\mathrm{BD}$ conditions in rats.

\section{Acknowledgements}

Not applicable.

\section{Funding}

The present study was supported by the China Postdoctoral Science Foundation (grant no. 2015M582209) and the Key Project of the Science and Technology Research Education Department of Henan Province (grant no. 16A320030).

\section{Availability of data and materials}

The datasets used during the current study are available from the corresponding author on reasonable request.

\section{Authors' contributions}

RZ designed research and wrote the paper; WG, HF, SC and BY performed the rat experiments; SC, KZ and SZ analyzed the data. All the authors approved the final manuscript.

\section{Ethics approval and consent to participate}

The Ethics Committee of the First Affiliated Hospital of Zhengzhou University (Zhengzhou, China) approved this study protocol.

\section{Consent for publication}

Not applicable.

\section{Competing interests}

The authors declare that they have no competing interests. 


\section{References}

1. Tacke F, Kroy DC, Barreiros AP and Neumann UP: Liver transplantation in Germany. Liver Transplant 22: 1136-1142, 2016.

2. Van der Hoeven JA, Lindell S, van Schilfgaarde R, Molema G, Ter Horst GJ, Southard JH and Ploeg RJ: Donor brain death reduces survival after transplantation in rat livers preserved for $20 \mathrm{hr}$. Transplantation 72: 1632-1636, 2001.

3. Neuberger J: Liver transplantation in the United Kingdom. Liver Transpl 22: 1129-1135, 2016.

4. Westendorp WH, Leuvenink HG and Ploeg RJ: Brain death induced renal injury. Curr Opin Organ Transplant 16: 151-156, 2011.

5. Pratschke J, Wilhelm MJ, Kusaka M, Basker M, Cooper DK, Hancock WW and Tilney NL: Brain death and its influence on donor organ quality and outcome after transplantation. Transplantation 67: 343-348, 1999.

6. Olinga P, van der Hoeven JA, Merema MT, Freund RL, Ploeg RJ and Groothuis GM: The influence of brain death on liver function. Liver Int 25: 109-116, 2005.

7. Novitzky D, Mi Z, Videla LA, Collins JF and Cooper DK: Thyroid hormone therapy and procurement of livers from brain-dead donors. Endocr Res 41: 270-273, 2016.

8. Leithead JA, Armstrong MJ, Corbett C, Andrew M, Kothari C, Gunson BK, Muiesan P and Ferguson JW: Hepatic ischemia reperfusion injury is associated with acute kidney injury following donation after brain death liver transplantation. Transpl Int 26: 1116-1125, 2013

9. Van Der Hoeven JA, Moshage H, Schuurs T, Nijboer M, Van Schilfgaarde R and Ploeg RJ: Brain death induces apoptosis in donor liver of the rat. Transplantation 76: 1150-1154, 2003.

10. Wen SW, Ager EI, Neo J and Christophi C: The renin angiotensin system regulates Kupffer cells in colorectal liver metastases. Cancer Biol Ther 14: 720-727, 2013.

11. Tsutsui $H$ and Nishiguchi S: Importance of Kupffer cells in the development of acute liver injuries in mice. Int J Mol Sci 15: 7711-7730, 2014.

12. Liu C, Yang Z, Wang L, Lu Y, Tang B, Miao H, Xu Q and Chen X: Combination of sorafenib and gadolinium chloride $(\mathrm{GdCl} 3)$ attenuates dimethylnitrosamine(DMN)-induced liver fibrosis in rats. BMC Gastroenterol 15: 159, 2015.

13. Cao S, Wang T, Yan B, Lu Y, Guo W and Zhang S: Protective effects of SP600125 in brain death-induced liver injury. Clin Res Hepatol Gastroenterol 38: 577-582, 2014.

14. Zhang S, Cao S, Wang T, Yan B, Lu Y and Zhao Y, Modified brain death model for rats. Exp Clin Transplant 12: 469-473, 2014.

15. Pratschke J, Wilhelm MJ, Kusaka M, Laskowski I and Tilney NL: A model of gradual onset brain death for transplant-associated studies in rats. Transplantation 69: 427-430, 2000.

16. Knodell RG, Ishak KG, Black WC, Chen TS, Craig R, Kaplowitz N, Kiernan TW and Wollman J: Formulation and application of a numerical scoring system for assessing histological activity in asymptomatic chronic active hepatitis. Hepatology 1: 431-435, 1981.

17. Desmet VJ: Knodell RG, Ishak KG, Black WC, Chen TS, Craig R, Kaplowitz N, Kiernan TW, Wollman J. Formulation and application of a numerical scoring system for assessing histological activity in asymptomatic chronic active hepatitis [Hepatology 1981;1:431-435]. J Hepatol 38: 382-386, 2003.

18. Wu Y, Wang Y, Li M, Yang X, Gong J and Zhang W: Gadolinium chloride suppresses acute rejection and induces tolerance following rat liver transplantation by inhibiting Kupffer-cell activation. Exp Ther Med 8: 1777-1782, 2014

19. Ellett JD, Atkinson C, Evans ZP, Amani Z, Balish E, Schmidt MG, van Rooijen N, Schnellmann RG and Chavin KD: Murine Kupffer cells are protective in total hepatic ischemia/reperfusion injury with bowel congestion through IL-10. J Immunol 184 5849-5858, 2010.
20. Barklin A: Systemic inflammation in the brain-dead organ donor. Acta Anaesthesiol Scand 53: 425-435, 2009.

21. Weiss S, Kotsch K, Francuski M, Reutzel-Selke A, Mantouvalou L, Klemz R, Kuecuek O, Jonas S, Wesslau C, Ulrich F, et al: Brain death activates donor organs and is associated with a worse $I / R$ injury after liver transplantation. Am J Transplant 7: 1584-1593, 2007.

22. Kotsch K, Ulrich F, Reutzel-Selke A, Pascher A, Faber W, Warnick P, Hoffman S, Francuski M, Kunert C, Kuecuek O, et al: Methylprednisolone therapy in deceased donors reduces inflammation in the donor liver and improves outcome after liver transplantation: A prospective randomized controlled trial. Ann Surg 248: 1042-1050, 2008.

23. Zhu C, Li J, Zhang G, Zhang Y, Zhai W, Shi J, Li Z, Li J and Zhang S: Brain death disrupts structure and function of pig liver. Transplant Proc 42: 733-736, 2010.

24. Kuecuek O, Mantouvalou L, Klemz R, Kotsch K, Volk HD, Jonas S, Wesslau C, Tullius S, Neuhaus P and Pratschke J: Significant reduction of proinflammatory cytokines by treatment of the brain-dead donor. Transplant Proc 37: 387-388, 2005

25. Li JQ, Qi HZ, He ZJ, Hu W, Si ZZ, Li YN and Li DB: Cytoprotective effects of human interleukin-10 gene transfer against necrosis and apoptosis induced by hepatic cold ischemia/reperfusion injury. J Surg Res 157: e71-e78, 2009.

26. Olinga P, Merema MT, de Jager MH, Derks F, Melgert BN, Moshage H, Slooff MJ, Meijer DK, Poelstra K and Groothuis GM: Rat liver slices as a tool to study LPS-induced inflammatory response in the liver. J Hepatol 35: 187-194, 2001

27. Cao S, Wang T, Yan B, Lu Y, Zhao Y and Zhang S: Brain death is associated with endoplasmic reticulum stress and apoptosis in rat liver. Transplant Proc 46: 3297-3302, 2014.

28. Baskin-Bey ES, Washburn K, Feng S, Oltersdorf T, Shapiro D, Huyghe M, Burgart L, Garrity-Park M, van Vilsteren FG, Oliver LK, et al: Clinical trial of the pan-caspase inhibitor, IDN-6556, in human liver preservation injury. Am J Transplant 7: 218-225, 2007

29. Sutter AG, Palanisamy AP, Ellet JD, Schmidt MG, Schnellmann RG and Chavin KD: Intereukin-10 and Kupffer cells protect steatotic mice livers from ischemia-reperfusion injury. Eur Cytokine Netw 25: 69-76, 2014.

30. Wan J, Benkdane M, Teixeira-Clerc F, Bonnafous S, Louvet A, Lafdil F, Pecker F, Tran A, Gual P, Mallat A, et al: M2 Kupffer cells promote M1 Kupffer cell apoptosis: A protective mechanism against alcoholic and nonalcoholic fatty liver disease. Hepatology 59: 130-142, 2014.

31. Wang B, Zhang Q, Zhu B, Cui Z and Zhou J: Protective effect of gadolinium chloride on early warm ischemia/reperfusion injury in rat bile duct during liver transplantation. PLoS One 8: e52743, 2013.

32. Golling M, Mehrabi A, Blum K, Jahnke C, Kellner H, Bud O, Hashemi B, Breitkreutz R, Becker-Brandenburg K, Schemmer P, et al: Effects of hemodynamic instability on brain death-induced prepreservation liver damage. Transplantation 75: 1154-1159, 2003.

33. Ritschl PV, Ashraf MI, Oberhuber R, Mellitzer V, Fabritius C, Resch T, Ebner S, Sauter M, Klingel K, Pratschke J and Kotsch K: Donor brain death leads to differential immune activation in solid organs but does not accelerate ischaemia-reperfusion injury. J Pathol 239: 84-96, 2016.

This work is licensed under a Creative Commons Attribution-NonCommercial-NoDerivatives 4.0 International (CC BY-NC-ND 4.0) License. 